\title{
Osteoprotegerin Level Differences in Orthodontic Treatment with Self-ligating and Conventional Preadjusted Brackets at Early Aligning and Leveling Phase
}

\author{
${ }^{1}$ Retno Widayati, ${ }^{2}$ Muhammad SK Adiwirya, ${ }^{3}$ Nurtami Soedarsono
}

\begin{abstract}
Aim: This study aims to investigate the differences in osteoprotegerin (OPG) concentrations in early aligning and leveling of orthodontic treatments using self-ligating and conventional preadjusted brackets.
\end{abstract}

Materials and methods: Three groups consisting of two experimental groups and one control group participated in this study. A total of 18 patients were selected according to the following inclusion criteria: Little's irregularity index on maxillary anterior teeth ranging from 4 to $9 \mathrm{~mm}$, nonextraction orthodontic treatment on the experimental group, and no previous orthodontic treatment. The experimental group received orthodontic treatment with either a passive self-ligating or conventional preadjusted bracket system, while the control group did not receive orthodontic treatment. Gingival crevicular fluid (GCF) collection was done at baseline, 1, 24, and 168 hours on five points of maxillary anterior proximal sites, from the mesiolabial side of the canine to the mesiolabial side of its contralateral side. The OPG concentration was assessed by enzyme-linked immunoabsorbent assay (ELISA).

Results: The OPG concentration on both bracket systems was decreased in a time-dependent manner. Decreasing concentration was maintained up until 168 hours on the selfligating bracket, while conventional preadjusted bracket showed significantly increasing concentration at 168 hours $(p=0.004)$. Levels of OPG were significantly lower on self-ligating bracket compared with the conventional bracket at 1, 24, and 168 hours $(p<0.005)$

Conclusion: Both bracket systems showed decreasing OPG concentration up to 24 hours after orthodontic activation. A maintained decreasing level of OPG was observed on the selfligating bracket group at 168 hours, and it showed significant lower OPG concentration compared with the conventional preadjusted bracket group at 1,24 , and 168 hours after initial force application

Clinical significance: The maintained decreasing level of OPG could indicate bone resorption that allows tooth movement into

1,2Department of Orthodontics, Faculty of Dentistry, Universitas Indonesia, Jakarta Pusat, DKI Jakarta, Indonesia

${ }^{3}$ Department of Oral Biology, Faculty of Dentistry, Universitas Indonesia, Jakarta Pusat, DKI Jakarta, Indonesia

Corresponding Author: Retno Widayati, Department of Orthodontics, Faculty of Dentistry, Universitas Indonesia, Jakarta Pusat, DKI Jakarta, Indonesia, Phone: +62811965364, e-mail: widayati22@yahoo.com correct alignment that still occurs on self-ligating bracket up to 168 hours after initial activation.

Keywords: Conventional preadjusted bracket, Orthodontic tooth movement osteoprotegerin, Self-ligating bracket.

How to cite this article: Widayati R, Adiwirya MSK, Soedarsono N Osteoprotegerin Level Differences in Orthodontic Treatment with Self-ligating and Conventional Preadjusted Brackets at Early Aligning and Leveling Phase. World J Dent 2018;9(1):2-7.

Source of support: Nil

Conflict of interest: None

\section{INTRODUCTION}

Orthodontic tooth movement is induced by the frequency and duration of mechanical stress, to which periodontal ligament tissues will respond. The mechanical orthodontic forces will be transmitted through the periodontal ligament to the alveolar bone, resulting in areas of tension or compression that lead to an inflammation process with various cellular reactions. ${ }^{1}$ Inflammatory mediators, such as chemokines, cytokines, and growth factors will be produced on both the tension and compression sides, which will activate tissue remodeling, including bone tissue. ${ }^{2,3}$ Bone remodeling is a dynamic interaction between bone-forming osteoblasts and bone-resorbing osteoclasts, which is an important event in orthodontic tooth movement. ${ }^{4}$

Cytokines are defined as extracellular signaling proteins directly involved during orthodontic tooth movement, which facilitate differentiation, activation, and apoptosis in bone and periodontal ligament cells. ${ }^{3}$ Their mechanisms of action during orthodontic tooth movement are classified as effector (proinflammatory) and suppressive (anti-inflammatory) cytokines. Some of the proinflammatory cytokines that are involved in osteoclastogenesis are the receptor activator of nuclear factor kappa-B ligand (RANKL) and the macrophage colony-stimulating factor (M-CSF), which are produced by osteoblasts and apoptotic osteocytes. The M-CSF will bind to its receptor, c-msf, and this process is required for progenitor cells to differentiate into osteoclasts, while also maintaining the survival and longevity of osteoclast precursors. ${ }^{5,6}$ The differentiation process of osteoclast 
precursors is unable to be completed by M-CSF on its own; it requires the expression of RANKL by osteoblastic stromal cells and of RANK by osteoclast precursors. ${ }^{5}$ The RANKL will exert its effect by binding to the RANK receptor on osteoclast lineage cells, which leads to rapid differentiation of the hematopoietic osteoclast precursors to mature osteoclasts. ${ }^{3}$

Bone remodeling is controlled by a balance between RANK-RANKL binding and OPG production. The OPG is a decoy receptor produced by osteoblastic cells, which competes with RANK for RANKL binding. On bone cells, OPG will inhibit the terminal stages of osteoclast differentiation, suppress matrix osteoclasts activation, and also induce apoptosis. ${ }^{3}$ In other words, a change in the RANKL/OPG ratio due to either an increase in the former or a decrease in the latter or a change in both will have an effect on osteoclast numbers and activity. ${ }^{5}$ The orthodontic forces will distort the extracellular matrix of the periodontal ligament and also alter the cellular shape and cytoskeletal configurations. These changes may modify GCF composition and flow rate. ${ }^{3}$ The GCF analysis may provide a noninvasive method to investigate the biochemical changes and cellular response of the underlying periodontal ligament during orthodontic movement. ${ }^{4,7}$ Studies have confirmed that both RANKL and OPG can be detected in human GCF during orthodontic movement. ${ }^{8-10}$

The methods of orthodontic treatment mechanics have been developed throughout orthodontics history to complement clinicians achieving the desired result on various orthodontic cases. ${ }^{11}$ As many studies have shown, there is an induction of cytokines, including OPG, during orthodontic movement using edgewise or preadjusted brackets. ${ }^{4,8-10,12}$ On the contrary, no studies have confirmed the level of OPG in GCF during orthodontic movement using self-ligating brackets. Furthermore, there is no detailed information about the comparison of OPG level changes during orthodontic treatment using preadjusted and self-ligating brackets. In the present study, we compared the levels of OPG in GCF samples during anterior crowding initial alignment at baseline, 1, 24, and 168 hours taken from orthodontic patients with preadjusted and self-ligating brackets.

\section{MATERIALS AND METHODS}

\section{Subject Selection}

The research protocol was reviewed and approved by the Ethical Committee of the Faculty of Dentistry, Universitas Indonesia (No. 12/Ethical Approval/FKGUI/III/2017). Two groups of orthodontic patients and one group of control subjects took part in this study. Informed consent was obtained from all subjects after they had received detailed information about the research. One group consisted of six orthodontic patients using preadjusted brackets ( 6 females, mean age $23 \pm 6.28$ years) and the other group consisted of six orthodontic patients using self-ligating brackets ( 3 males, 3 females, mean age $25.5 \pm$ 4.91 years). Six subjects without orthodontic treatment were appointed as the control group ( 3 males, 3 females, mean age $26.67 \pm 6.83$ years), which has the same criteria as the orthodontic patients group. The subjects were selected to participate in this study based on the following criteria: (1) Good general health and healthy periodontal tissue, with probing depths not exceeding $3 \mathrm{~mm}$, (2) no sign of bone loss in panoramic radiograph, (3) no history of antibiotic therapy within the past 6 months, (4) not under anti-inflammatory drugs in the month preceding the start of the study, (5) Little's irregularity index ${ }^{13}$ on maxillary anterior ranging from 4 to $9 \mathrm{~mm}$, (6) nonextraction orthodontic treatment on the experimental group, and (7) never had previous orthodontic treatment.

\section{Experimental Design}

Two experimental groups were set up, group I treated with preadjusted brackets (MBT, Ormco, Glendora, California, USA) and group II treated with passive selfligating brackets (Damon Q, Ormco, Glendora, California, USA). Maxillary arch impressions were taken on all subjects to measure the Little's irregularity index. Both experimental groups were orthodontically treated during the initial leveling and alignment for 168 hours beginning with 0.014-inch nickel-titanium archwires (Dentsply GAC, Bohemia, New York, USA) for group I. Meanwhile, group II began with 0.014-inch copper nickel-titanium archwires (Ormco, Glendora, California, USA). The archwires for group I were attached to the brackets using elastomeric ligatures. Maxillary anterior brackets must be engaged in the initial bracket bonding and they were not allowed to be removed during the study. The control group was not treated with orthodontic treatment. A session of supragingival scaling and oral hygiene maintenance instruction was given to all subjects to achieve good plaque control. All subjects were warned not to take any of the anti-inflammatory drugs during the period of study.

\section{Gingival Crevicular Fluid Collection}

At the time of each visit for GCF collection, the oral hygiene index was assessed using the simplified oral hygiene index by Greene and Vermillion. ${ }^{14}$ The GCF was collected before activation and at the following time periods after the activation: 1, 24, and 168 hours at five points of maxillary anterior proximal sites, from the mesiolabial side of the canine to the mesiolabial side of 
its contralateral. Small deposits of plaque were removed with a periodontal probe without touching the gingiva to minimize the contamination of the GCF samples. The six maxillary anterior teeth were gently washed with water, isolated with cotton rolls, and then gently dried with an air syringe. One paper point was used for each site undergoing examination using the method of Offenbacher et $\mathrm{al}^{15}$ to collect GCF samples. Paper points (ISO 30, Gapadent, Tianjin, PR, China) were carefully inserted $1 \mathrm{~mm}$ into the gingival crevice on each site and allowed to remain for 30 seconds. The insertion of paper points was done twice in each site, with a 1-minute interval between collections. Any paper points visibly contaminated with blood were discarded. Paper points were then placed into a microcentrifuge tube filled with $500 \mu \mathrm{L}$ of phosphate-buffered saline and stored at $-80^{\circ} \mathrm{C}$ before laboratory analysis.

\section{Total Protein Concentration and OPG Determination}

All samples were thawed and subjected to a vortex for 30 seconds. The GCF from samples was then eluted from paper points by centrifugal filtration at $1,000 \times \mathrm{g}$ for 20 minutes at $4^{\circ} \mathrm{C}$. After being centrifuged, the paper points were removed and the supernatants were transferred into a new microcentrifuge tube. Total protein concentration in the extract was estimated by Bradford ${ }^{16}$ protein assay, with bovine serum albumin as a standard. The extracts were standardized by diluting them to the concentration of $50 \mu \mathrm{g} / \mathrm{mL}$ before being processed on the ELISA. The OPG was measured by sandwich ELISA kit (Elabscience, Human OPG, Wuhan, PR China). The assays were carried out in duplicate both for reference standards and samples in accordance with the manufacturer's instructions. Total level of OPG measurement present in GCF samples was recorded as $\mathrm{ng} / \mathrm{mL}$ derived from the protein standard curve.

\section{Statistical Methods}

Values were determined as the mean \pm standard deviation. The data were processed and analyzed using
Statistical Package for the Social Sciences version 21 for Mac (IBM SPSS Statistics, Illinois, USA). The OPG level distributions $(\mathrm{ng} / \mathrm{mL})$ were examined using the Shapiro-Wilk's normality test and the comparisons were performed using parametric tests. A two-way repeated measures analysis of variance (ANOVA) was used to investigate differences in OPG levels throughout experimental periods on all groups. The differences in OPG concentration among the groups at specific time points were tested by one-way ANOVA.

\section{RESULTS}

The purpose of this study was to evaluate the OPG level changes $(\mathrm{ng} / \mathrm{mL})$ during an early stage of orthodontic treatment with self-ligating and conventional preadjusted brackets. The OPG level changes of each bracket system and control subject throughout the experimental periods are shown in Table 1 . The OPG levels were decreased on both the self-ligating and the conventional preadjusted group up to the 24-hour experimental period. The level of OPG was increased at 168 hours on the conventional preadjusted group, approaching its baseline value. On the contrary, the OPG level on the self-ligating bracket continued to decrease at 168 hours. The changes of OPG concentration on the self-ligating bracket were statistically significant among the experimental periods, with the exception of the decreased level of OPG at 24 to 168 hours. In addition, the statistically significant changes of OPG level were noticed for the conventional preadjusted group at 1 to 24 hours and also 24 to 168 hours. The control group showed a slight rise and fall of OPG concentration, but no statistically significant change was detected throughout the experimental periods. Time-dependent changes of OPG level on all groups are shown in Graph 1.

The mean of the OPG levels among the self-ligating bracket, conventional preadjusted bracket, and control group at specific experimental periods are compared in Table 2. None of the groups showed a statistically significant difference of OPG levels at baseline. Conversely, significant differences were observed among

Table 1: The OPG level longitudinal changes throughout experimental periods in orthodontically treated groups together with control group

\begin{tabular}{|c|c|c|c|c|c|c|c|c|c|c|c|}
\hline \multirow[b]{2}{*}{ Group } & \multirow[b]{2}{*}{$n$} & \multicolumn{4}{|c|}{ Mean of OPG levels (SD) in $\mathrm{ng} / \mathrm{mL}^{*}$} & \multicolumn{6}{|c|}{$p$-values } \\
\hline & & TO (baseline) & T1 (1 hour) & T2 (24 hours) & T3 (168 hours) & $\begin{array}{l}\text { TO vs } \\
\text { T1 }\end{array}$ & $\begin{array}{l}\text { T0 vs } \\
\text { T2 }\end{array}$ & $\begin{array}{l}\text { T0 vs } \\
\text { T3 }\end{array}$ & $\begin{array}{l}\text { T1 vs } \\
\text { T2 }\end{array}$ & $\begin{array}{l}\text { T1 vs } \\
\text { T3 }\end{array}$ & $\begin{array}{l}\text { T2 vs } \\
\text { T3 }\end{array}$ \\
\hline $\begin{array}{l}\text { Preadjusted } \\
\text { bracket }\end{array}$ & 6 & $0.954(0.095)$ & $0.804(0.075)$ & $0.503(0.099)$ & $0.811(0.071)$ & 0.15 & $0.001^{*}$ & 0.192 & $0.006^{*}$ & 1.000 & $0.004^{*}$ \\
\hline $\begin{array}{l}\text { Self-ligating } \\
\text { bracket }\end{array}$ & 6 & $1.016(0.065)$ & $0.641(0.371)$ & $0.223(0.027)$ & $0.196(0.031)$ & $0^{*}$ & $0^{*}$ & $0^{*}$ & $0^{*}$ & $0^{*}$ & 1.000 \\
\hline Control & 6 & $1.046(0.153)$ & $1.099(0.155)$ & $0.963(0.105)$ & $0.975(0.131)$ & 1.000 & 1.000 & 1.000 & 0.923 & 0.783 & 1.000 \\
\hline
\end{tabular}

*Statistically significant difference at $p<0.05$, pairwise comparisons Bonferroni post hoc test; SD: Standard deviation 


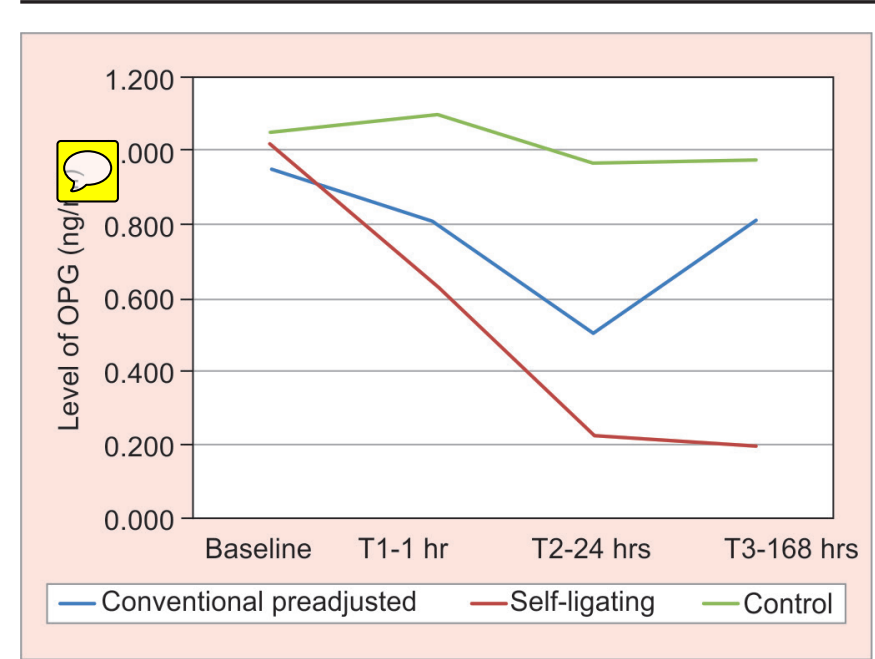

Graph 1: Osteoprotegerin level in time-dependent changes in GCF from 1 to 168 hours after orthodontic activation

the groups at 1 and 24 hours experimental period. At 168 hours experimental period, statistically significant differences were also noticed between the preadjusted and self-ligating brackets together with the self-ligating bracket and control group.

\section{DISCUSSION}

The RANKL/RANK/OPG system has been thought as the most important advanced finding in bone biology in the last decade. ${ }^{5}$ The discovery of this system also plays an important role in orthodontic tooth movement researches. Many studies have investigated about OPG expression in the GCF during orthodontic movement, mostly in a canine undergoing distal movement. ${ }^{4,8-10,12}$ In the current study, changes in OPG levels among orthodontic patients with different bracket systems were assessed during the early aligning and leveling phases. Differences in the force systems of different bracket systems will be evaluated through differences of OPG level contained in GCF.

We found decreased levels of OPG in GCF samples through 1 up to 24 hours on both conventional preadjusted and self-ligating systems. Interestingly, the OPG level started to increase approaching its baseline value at 168 hours on the conventional preadjusted bracket, while the self-ligating bracket continued to show decreasing OPG concentration even more. Conversely, the group without orthodontic treatment did not show any differences of OPG level throughout the entire experimental period. Thus, its OPG level is appropriate to be assigned as a control. Our findings on the conventional brackets group are supported by Nishijima et $\mathrm{al}^{8}$ and Kawasaki et al. ${ }^{9}$ They determined a similar pattern of OPG expression in orthodontic movement within 24 hours after initial activation, which is the lowest OPG level among the experimental periods and this began to increase by 168 hours. However, the orthodontic movement and type of bracket used from those two studies were different from our current study. Those studies investigated OPG expression during the 1-week period of canine distalization using an elastomeric chain on an edgewise bracket, with GCF collected at the distal aspect of the canine or the compression side.

The decreasing level of OPG up to 168 hours on our self-ligating bracket group is somewhat supported by Toygar et al. ${ }^{4}$ The study showed that OPG decreased significantly at the 1 hour and was maintained at 24 , 168 hours, 1 and 3 months after orthodontic activation. Similar results also can be observed in a study by Grant et $\mathrm{al}_{1}^{10}$ which showed decreasing levels of OPG concentration up to 42 days after force application. Again, there are differences with our current study in terms of study design, bracket system, force magnitude, and also local response of bone or age differences. However, the continuous force being exerted from a closed superelastic nitinol coil spring in those two studies can be considered comparable with the continuous force applied on the selfligating bracket system. The term "self-ligating bracket" is used for brackets that incorporate a locking mechanism that holds the archwire in the bracket slot. ${ }^{17}$ We use a passive self-ligating bracket type in our study, which was considered to have lower friction between bracket and archwire and also more net effective force properties compared with conventional brackets. ${ }^{18}$ Friction in orthodontic treatment is viewed as a force that must be overcome before the intended tooth-moving forces will take their effect and this relative movement between the

Table 2: Comparison of OPG level mean changes among orthodontically treated and control group at a specific experimental period

\begin{tabular}{|c|c|c|c|c|c|c|c|}
\hline \multirow[b]{2}{*}{ Time periods } & \multirow[b]{2}{*}{$n$} & \multicolumn{3}{|c|}{ Mean of OPG levels (SD) in $n g / \mathrm{mL}^{*}$} & \multicolumn{3}{|c|}{$p$-values } \\
\hline & & $\begin{array}{l}\text { Preadjusted } \\
\text { bracket }\end{array}$ & $\begin{array}{l}\text { Self-ligating } \\
\text { bracket }\end{array}$ & Control & $\begin{array}{l}\text { Preadjusted bracket } \\
\text { vs self-ligating bracket }\end{array}$ & $\begin{array}{l}\text { Preadjusted } \\
\text { bracket vs control }\end{array}$ & $\begin{array}{l}\text { Self-ligating } \\
\text { bracket vs control }\end{array}$ \\
\hline T0 (baseline) & 6 & $0.954(0.095)$ & $1.016(0.065)$ & $1.046(0.153)$ & 1.000 & 0.512 & 1.000 \\
\hline T1 (1 hour) & 6 & $0.804(0.075)$ & $0.641(0.371)$ & $1.099(0.155)$ & $0.005^{\star}$ & $0.011^{*}$ & $0.002^{*}$ \\
\hline T2 (24 hours) & 6 & $0.503(0.099)$ & $0.223(0.027)$ & $0.963(0.105)$ & $0.002^{*}$ & $0^{*}$ & $0^{*}$ \\
\hline T3 (168 hours) & 6 & $0.811(0.071)$ & $0.196(0.031)$ & $0.975(0.131)$ & 9 & 0.083 & $0^{*}$ \\
\hline
\end{tabular}

*Statistically significant difference at $p<0.05$, Bonferroni (baseline) drut Tamhane T2 (T1, T2, T3) post hoc test; SD: Standard deviation 
bracket and the archwire can occur. Baccetti et $\mathrm{al}^{19}$ and Higa et $\mathrm{al}^{20}$ have investigated the net effective force on passive self-ligating brackets. Those studies found the amount of force for correction in vertical and buccolingual misalignment is generated higher on the passive self-ligating bracket compared with the conventional bracket at the same degree of teeth displacement.

We propose that differences of friction and net effective force properties between different system brackets can explain this phenomenon. At the same degree of teeth displacement, represented by a similar Little's irregularity index between the groups in our study, lower friction will allow more net affective force that could be transmitted through the periodontal ligament to the alveolar bone, leading to bone remodeling. The force differences are reflected in the GCF composition, with the OPG level found to be significantly lower on the self-ligating bracket compared with the conventional preadjusted bracket group at 1,24, and also 168 hours. In bone remodeling, OPG serves as a decoy receptor for RANKL, thereby inhibiting osteoclast differentiation. The maintained decreasing level of OPG up to 168 hours on our self-ligating bracket group could indicate that bone resorption allowing tooth movement into correct alignment was still occurring during the final experimental period. In addition, the insignificant decreasing change of OPG level from 24 to 168 hours on the self-ligating bracket presumably indicates that this bracket system still exerts light continuous force to allow more tooth movement.

On the contrary, the OPG concentration on the conventional preadjusted bracket group started to increase approaching its baseline value at 168 hours, which indicates less bone resorption than the self-ligating bracket group at that specific time period. This could have happened due to aging of the elastic ligatures as stated by Guimarães et al..$^{21}$ The orthodontic elastomers, which were used on our conventional bracket group, were biodegraded in the oral environment by hydrolyses, which leads to relaxation of the elastomers. The consequence of the relaxation phenomenon is a decrease on the mechanical energy transmitted to the tooth resulting in reduction of tooth movement effectiveness. In that study, orthodontic elastomers already showed degradation at the 1-week placement in the oral environment, as evaluated by scanning electron microscopy.

The changes in OPG level by the analysis of GCF may contribute to a better understanding of cellular activities, especially in bone, related to orthodontic movement. Our present study is the first in vivo study that points out differences of OPG concentration between different bracket systems used during the early aligning and leveling phase of orthodontic treatment. Extensive research is needed to understand more about further changes of the OPG level during the long-term phases of orthodontic tooth movement on self-ligating and conventional preadjusted bracket systems.

\section{CONCLUSION}

- Both bracket systems showed decreasing OPG concentration up to 24 hours after orthodontic activation.

- The level of OPG on the self-ligating bracket group continued decreasing at 168 hours, while in the conventional preadjusted bracket group, it started to increase approaching its baseline value during the same time period.

- The self-ligating bracket group showed significantly lower OPG concentration at 1, 24, and 168 hours after initial activation compared with the conventional preadjusted bracket group.

\section{CLINICAL SIGNIFICANCE}

The maintained decreasing level of OPG could indicate that bone resorption process still occurs on self-ligating bracket group up to 168 hours after initial activation. Optimum orthodontic tooth movement into correct alignment could be expected more to happen on self-ligating bracket group during experimental period.

\section{ACKNOWLEDGMENT}

Authors would like to thank the Research and Community Service Directorate of Universitas Indonesia for supporting this research through 2017 PITTA-UI grant.

\section{REFERENCES}

1. Krishnan V, Davidovitch, Z. Biology of orthodontic tooth movement: the evolution of hypotheses and concepts. In: Krishnan V, Davidovitch Z, editors. Biological mechanisms of tooth movement. 2nd ed. Ames (IA): John Wiley \& Sons, Ltd.; 2015. pp. 15-29.

2. Andrade I Jr, Taddei SR, Souza PE. Inflammation and tooth movement: the role of cytokines, chemokines, and growth factors. Semin Orthod 2012 Dec;18(4):257-269.

3. Krishnan V, Davidovitch Z. Cellular, molecular, and tissuelevel reactions to orthodontic force. Am J Orthod Dentofacial Orthop 2006 Apr;129(4):469.e1-469.e32.

4. Toygar HU, Kircelli BH, Bulut S, Sezgin N, Tasdelen B. Osteoprotegerin in gingival crevicular fluid under long-term continuous orthodontic force application. Angle Orthod 2008 Nov;78(6):988-993.

5. Boyce BF, Xing L. Functions of RANKL/RANK/OPG in bone modeling and remodeling. Arch Biochem Biophys 2008 May;473(2):139-146.

6. Kitaura H, Zhou P, Kim HJ, Novack DV, Ross FP, Teitelbaum SL. M-CSF mediates TNF-induced inflammatory osteolysis. J Clin Invest 2005 Dec;115(12):3418-3427.

7. Alhadlaq AM. Biomarkers of orthodontic tooth movement in gingival crevicular fluid: a Systematic review. J Contemp Dent Pract 2015 Jul;16(7):578-587.

8. Nishijima Y, Yamaguchi M, Kojima T, Aihara N, Nakajima R, Kasai K. Levels of RANKL and OPG in gingival crevicular 
fluid during orthodontic tooth movement and effect of compression force on releases from periodontal ligament cells in vitro. Orthod Craniofac Res 2006 May;9(2):63-70.

9. Kawasaki K, Takahashi T, Yamaguchi M, Kasai K. Effects of aging on RANKL and OPG levels in gingival crevicular fluid during orthodontic tooth movement. Orthod Craniofac Res 2006 Aug;9(3):137-142.

10. Grant M, Wilson J, Rock P, Chapple I. Induction of cytokines, MMP9, TIMPs, RANKL and OPG during orthodontic tooth movement. Eur J Orthod 2013 Oct;35(5):644-651.

11. McLaughlin RP, Bennett JC. Evolution of treatment mechanics and contemporary appliance design in orthodontics: a 40-year perspective. Am J Orthod Dentofacial Orthop 2015 Jun;147(6):654-662.

12. Tuncer BB, Ozdemir BC, Boynuegri D, Karakaya IB, Erguder U, Yucel AA, Aral LA, Ozmeric N. OPG-RANKL levels after continuous orthodontic force. Gazi Med J 2013 Apr;24(2): 33-36.

13. Burns A, Dowling AH, Garvey TM, Fleming GJ. The reliability of little's irregularity index for the upper dental arch using three dimensional (3D) digital models. J Dent 2014 Oct;42(10):1320-1326.

14. Greene JC, Vermillion JR. The simplified oral hygiene index. The Journal of The American Dental Association. 1964; 68:7-13.
15. Offenbacher S, Odle BM, Van Dyke TE. The use of crevicular fluid prostaglandin E2 levels as a predictor of periodontal attachment loss. J Periodontal Res 1986 Mar;21(2):101-112.

16. Bradford MM. A rapid and sensitive method for the quantitation of microgram quantities of protein utilizing the principle of protein-dye binding. Anal Biochem 1976 May;72: 248-254.

17. Bock F. The development and history of fixed appliances. In: Ludwig B, Bister D, Baumgaertel S, editors. Self-ligating brackets in orthodontics: current concepts and techniques. New York: Thieme; 2012. pp. 2-6.

18. Harradine N. The history and development of self-ligating brackets. Semin Orthod 2008 Mar;14(1):5-18.

19. Baccetti T, Franchi L, Camporesi M, Defraia E, Barbato E. Forces produced by different nonconventional bracket or ligature systems during alignment of apically displaced teeth. Angle Orthod 2009 May;79(3):533-539.

20. Higa RH, Semenara NT, Henriques JF, Janson G, Sathler R, Fernandes TM. Evaluation of force released by deflection of orthodontic wires in conventional and self-ligating brackets. Dent Press J Orthod 2016 Nov-Dec;21(6):91-97.

21. Guimarães GS, de Morais LS, de Souza MM, Elias CN. Superficial morphology and mechanical properties of in vivo aged orthodontic ligatures. Dental Press J Orthod 2013 May-Jun;18(3):107-112. 